\section{The Odometry Error of a Mobile Robot With a Synchronous Drive System}

Agostino Martinelli

\begin{abstract}
This paper presents an error modeling of an odometry system for a synchronous-drive system and a possible strategy for evaluating this error. The odometry error is modeled by introducing four parameters characterizing its systematic and nonsystematic components (translational and rotational). The nonsystematic errors are expressed in terms of a covariance matrix, which depends on both the previous four parameters and the path followed by the mobile robot. In contrast to previous approaches which require assuming a particular path (straight or circular) in order to compute this covariance matrix, here general formulas are derived. We suggest a possible strategy for simultaneously estimating the four model parameters. As we will show, our strategy only requires measuring the change in the orientation and position between the initial and final configurations of the robot, related to suitable robot motions. In other words, it is unnecessary to know the actual path followed by the robot. We illustrate the proposed strategy by discussing the accuracy of the parameters estimation and by showing some experimental results obtained with the mobile robot Nomad150.
\end{abstract}

Index Terms-Localization, odometry, robot navigation.

\section{INTRODUCTION}

Determining the odometry errors of a mobile robot is very important, both in order to reduce them, and to know the accuracy of the state configuration estimated by using encoder data. The odometry error contains both systematic and nonsystematic components. Both components depend on the interaction of the robot with the environment where the robot moves. In particular, the nonsystematic component dramatically depends on the environment.

In a series of papers, Borenstein and collaborators [1]-[6], [21] investigated possible sources of both kinds of errors. A review of these sources is given in [6].

Regarding the systematic errors in differential-drive mobile robots, there are two dominant error sources, unequal wheel diameters and uncertainty about the effective wheel base. In Borenstein and Feng [5], a calibration technique called, "UMBmark" has been developed to calibrate systematic errors of a two-wheeled robot. This method has been used by other authors [8].

Goel et al. [11] used another calibration procedure to compensate for systematic errors. They referred to the differential-drive mobile robot Pioneer AT. They measured (when the robot was sitting on a box and the wheels rotated freely in the air) the actual velocities of the wheels and the velocity measurements from the encoders. In this way, they found a relationship between the velocity returned by the encoders and the actual velocity (measured by using a precise tachometer). Moreover,

Manuscript received July 25, 2001; revised February 5, 2002. This paper was recommended for publication by Associate Editor D. Kriegman and Editor A. DeLuca upon evaluation of the reviewers' comments. This work was supported by the Italian Ministry for Education, University, and Research, Project PRASSI, under "Calcolo Parallelo con Applicazioni alla Robotica" (Parallel Computing for Robotic Applications). This paper was presented at the 5th IFAC Symposium on Nonlinear Control Systems, St. Petersburg, Russia, July 4-6, 2001, and at the International Conference on Intelligent Robots and Systems, Maui, HI, October 29-November 3, 2001.

The author is with the Dipartimento di Informatica, Sistemi e Produzione, Università degli Studi di Roma "Tor Vergata," 00133 Rome, Italy (e-mail ago@robot2.disp.uniroma2.it).

Publisher Item Identifier S 1042-296X(02)05282-5. they measured the effective axle length due to skid steering which differs from that given by the specifications for the robot.

Finally, Roy and Thrun [19] suggested an algorithm that uses the robot's sensors to automatically calibrate the robot as it operates.

In a series of papers, Borenstein ([6] and references therein) also suggested a method to improve the accuracy of the odometry data by reducing the effect of the nonsystematic errors. With this method, called Internal Position Error Correction (IPEC), it was possible to detect and correct odometry errors without inertial or external-reference sensors. In particular, he implemented the IPEC method on the specially designed mobile robot platforms Multi Degree of Freedom (MDOF) [2] and Omnimate [6]. Experimental results showed that the accuracy achieved with the IPEC method was one to two orders of magnitude better than that of systems based on conventional dead reckoning.

Wang [20] analyzed the nonsystematic errors from a theoretical point of view, and computed the odometry error covariance matrix $Q$. He referred to a differential-drive mobile robot. In order to evaluate this matrix, he divided the entire path in $N$ small elementary paths. To compute the covariance matrix, he had to make some assumptions about the type of elementary path. In particular, he assumed a circular path. Moreover, since the updated robot position depended nonlinearly on changes in the translation and orientation (measured by the encoders), he had to introduce another approximation. He called the nonlinear term appearing in the updated position the adjustment factor. He analyzed three different cases, depending on the considered approximation for this factor. In particular, he considered a Taylor approximation of the adjustment factor truncated at the zero and first orders. Finally, as a third case, he considered this factor as a constant in the calculation of the covariance matrix.

The same approximations were made by Chenavier and Crowley [7] and by Feng and Milios [13]. They always considered a particular path and they used a Taylor approximation to compute the covariance matrix.

Chong and Kleeman [8] divided the entire path in $N$ small segments. They found, for the first time, a closed-form solution for the covariance matrix $Q$ as $N$ approaches infinity. In this way, they did not require the Taylor approximation. However, with their method, they were able to compute this matrix only for special cases. Their expressions were applicable to circular arc motions with constant radii of curvature, and included the limit cases of an infinity radius (straight motion) and zero radius (rotation about the center of the wheel axis).

Kelly [12] presented the general solution for linearized systematic error propagation for any trajectory and any error model.

Martinelli [14] derived general formulas for the covariance matrix. In these formulas, applicable to any path, the trajectory of the robot motion appeared as a function of the curve length. In these formulas, there were four parameters which depended on the robot and on the environment where the robot moved. Two parameters characterized the two nonsystematic components (translational and rotational). The other two parameters characterized the translational and rotational systematic components. Moreover, in [15] and [16], he suggested a strategy for estimating those parameters.

In this paper, we present both an error modeling of an odometry system for a synchronous-drive system and a possible strategy for evaluating this error. The odometry error model is discussed in Section II, where we introduce four parameters characterizing the elementary components of the odometry error (translational and rotational), both systematic and nonsystematic. In Section III, we express the nonsystematic components of the odometry error in terms of a covariance matrix, and we derive the general form of this matrix applicable to any robot motion. In Section IV, we introduce seven 


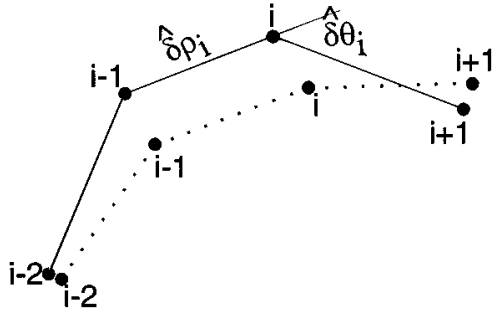

Fig. 1. Solid line represents the real motion followed by the robot when $N \rightarrow$ $\infty$, while the dotted line is the motion trajectory measured by the encoder.

experimentally measurable quantities for a given robot motion, which we call the observables. On the basis of our odometry error model, we analytically compute the mean values and the variances of the observables, which depend on the previous four parameters and on the considered robot motion. In Section V, we explicitly compute the observables for a simple robot motion. Evaluating the observables for this robot motion is a possible strategy for simultaneously estimating both systematic and nonsystematic parameters. This strategy only requires measuring the change in the orientation and position between the initial and final configurations of the robot, related to the considered robot motion. In other words, it does not require knowing the actual path followed by the robot. The proposed strategy is illustrated in Section VI, where the accuracy of the parameters estimation is discussed, and some experimental results obtained with the mobile robot Nomad150 are presented.

\section{OdOMETRY ERROR MODEL}

We consider a mobile robot with a synchronous drive system. Assuming a two-dimensional world, we can define the robot configuration with respect to a world-coordinate frame $W$ by the vector $X=$ $[x, y, \theta]^{T}$, containing its position and orientation. The robot configuration estimated by odometry measurements is different from the actual configuration $X$ because of the odometry errors.

In order to compute the global odometry error related to a given robot motion, we divided the trajectory in $N$ small segments (see Fig. 1). We first modeled the elementary error related to a single segment. Then we computed (Sections III-VI) the cumulative error on the global path. Finally, we took the limit value when $N \rightarrow \infty$.

We introduced the following assumptions about the actual motion.

1) The robot moves straight along each given segment whose length, measured by the encoder sensor, is always $\overline{\delta \rho}=\bar{\rho} / N$.

2) The angle $\widehat{\delta} \theta_{i}$ between the orientations related to the $(i+1)$ th and the $i$ th segment and the translation $\widehat{\delta} \rho_{i}$ covered during the same step are Gaussian random variables.

3) The random variable $\widehat{\delta} \rho_{i}$ is independent of the random variable $\widehat{\delta} \theta_{i}$, moreover, $\widehat{\delta} \rho_{i}$ is independent of $\widehat{\delta} \rho_{j}(i \neq j)$ and $\widehat{\delta} \theta_{i}$ is independent of $\widehat{\delta} \theta_{j}$.

We therefore can write

$$
\begin{aligned}
& \widehat{\delta} \rho_{i} \sim N\left(\overline{\delta \rho}\left(1+E_{T}\right), \sigma_{\delta \rho}^{2}\right) \\
& \widehat{\delta} \theta_{i} \sim N\left(\overline{\delta \theta_{i}}+E_{R} \overline{\delta \rho}, \sigma_{\delta \theta}^{2}\right)
\end{aligned}
$$

where $\overline{\delta \theta_{i}}$ is the angle between the orientations related to the $(i+1)$ th and the $i$ th segment measured by the encoder sensor, $E_{T} \overline{\delta \rho}$ and $E_{R} \overline{\delta \rho}$ represent the systematic components of the error, and $\sigma_{\delta \theta}^{2}$ and $\sigma_{\delta \rho}^{2}$ are directly related to the rolling conditions and are assumed to increase linearly with the traveled distance, i.e.,

$$
\sigma_{\theta}^{2}=K_{\theta} \bar{\rho}
$$

and

$$
\sigma_{\rho}^{2}=K_{\rho} \bar{\rho}
$$

The odometry error model here proposed is based on four parameters. Two of them $\left(E_{R}, E_{T}\right)$ characterize the systematic components, while the other two $\left(K_{\theta}, K_{\rho}\right)$ characterize the nonsystematic components. Clearly, these parameters depend on the environment where the robot moves. The assumption that $\widehat{\delta} \rho_{i}$ is independent of $\widehat{\delta} \theta_{i}$ is clearly a simplified approximation. A disturbance on the robot trajectory can generate both a distance error and a dependent-angle error. Moreover, more sophisticated models should also take into account the error dependence on the robot velocity and acceleration. Finally, we want to remark that a statistical treatment of the nonsystematic component assumes the environment homogeneous on large scale. Therefore, the expressions we are deriving in Sections III-VI hold. If the robot moves in regions larger than the scale beyond it, the environment can be considered homogeneous.

\section{COVARIANCE MATRIX $Q$}

The nonsystematic errors are expressed in terms of the covariance matrix $Q$. The robot configuration $X$ is a random vector whose mean value $\langle X\rangle$ is given by the odometry measurements (once both the systematic errors are known). The covariance matrix $Q$ is defined as

$$
Q=E\left\{[X-\langle X\rangle][X-\langle X\rangle]^{T}\right\}=\left[\begin{array}{ccc}
\sigma_{x}^{2} & \sigma_{x y} & \sigma_{x \theta} \\
\sigma_{x y} & \sigma_{y}^{2} & \sigma_{y \theta} \\
\sigma_{x \theta} & \sigma_{y \theta} & \sigma_{\theta}^{2}
\end{array}\right]
$$

This matrix, of course, depends on the trajectory followed by the robot, and also on the type of floor surface. It can be represented as a function of the previous parameters $\left(E_{R}, E_{T}, K_{\theta}\right.$, and $\left.K_{\rho}\right)$, which can be determined experimentally as suggested in Sections IV-VI. The analytical expression of the matrix entries, in terms of the model parameters and the trajectory, can be obtained through direct computation on the basis of the hypothesis stated in Section II. This computation is troublesome, with the exception of the term $\sigma_{\theta}^{2}$ directly given by (3). For $\sigma_{x}^{2}, \sigma_{y}^{2}$, and $\sigma_{x y}$ we use the relations $\sigma_{x}^{2}=\left\langle x^{2}\right\rangle-\langle x\rangle^{2}$, $\sigma_{y}^{2}=\left\langle y^{2}\right\rangle-\langle y\rangle^{2}$, and $\sigma_{x y}=\langle x y\rangle-\langle x\rangle\langle y\rangle$. Therefore, in order to compute the other matrix entries, we need to compute $\langle x\rangle,\langle y\rangle,\left\langle x^{2}\right\rangle$, $\left\langle y^{2}\right\rangle,\langle x y\rangle, \sigma_{x \theta}$, and $\sigma_{y \theta}$.

In Appendix I, we only give the computation of the mean value $\langle x\rangle$, obtaining

$$
\langle x\rangle=\left(1+E_{T}\right) \int_{0}^{\bar{\rho}} \cos (\widetilde{\theta}(s)) e^{-\left(K_{\theta^{s}} / 2\right)} d s
$$

where $\widetilde{\theta}(s)=\theta(s)+E_{R} s$ and $\theta(s)$ is the robot orientation as measured by the encoder sensor as a function of the curve length $s$ always measured by encoder. In a similar way the other previous quantities can be derived. We obtain

$$
\begin{aligned}
\langle y\rangle= & \left(1+E_{T}\right) \int_{0}^{\bar{\rho}} \sin (\tilde{\theta}(s)) e^{-K_{\theta} s / 2} d s \\
\left\langle x^{2}\right\rangle= & \left(1+E_{T}\right)^{2} \int_{0}^{\bar{\rho}} d s \int_{0}^{\bar{\rho}-s} d s^{\prime}\left\{e^{-K_{\theta} s^{\prime} / 2}\right. \\
& \times\left[\left(1+\chi_{C}(s)\right) \cos \left[\tilde{\theta}\left(s+s^{\prime}\right)-\tilde{\theta}(s)\right]\right. \\
& \left.\left. \pm \chi_{S}(s) \sin \left[\tilde{\theta}\left(s+s^{\prime}\right)-\tilde{\theta}(s)\right]\right]\right\} \\
& +\frac{K_{\rho}}{2}\left[\bar{\rho}+\int_{0}^{\bar{\rho}} \chi_{C}(s) d s\right]
\end{aligned}
$$




$$
\begin{aligned}
\left\langle y^{2}\right\rangle= & \left(1+E_{T}\right)^{2} \int_{0}^{\bar{\rho}} d s \int_{0}^{\bar{\rho}-s} d s^{\prime}\left\{e^{-K_{\theta s^{\prime} / 2}}\right. \\
& \times\left[\left(1-\chi_{C}(s)\right) \cos \left[\tilde{\theta}\left(s+s^{\prime}\right)-\tilde{\theta}(s)\right]\right. \\
& \left.\left.+\chi_{S}(s) \sin \left[\tilde{\theta}\left(s+s^{\prime}\right)-\tilde{\theta}(s)\right]\right]\right\} \\
& +\frac{K_{\rho}}{2}\left[\bar{\rho}+\int_{0}^{\bar{\rho}} \chi_{C}(s) d s\right] \\
\langle x y\rangle= & \left(1+E_{T}\right)^{2} \int_{0}^{\bar{\rho}} d s \int_{0}^{\bar{\rho}-s} d s^{\prime}\left\{e^{-K_{\theta} s^{\prime} / 2}\right. \\
& \times\left[\chi_{S}(s) \cos \left[\tilde{\theta}\left(s+s^{\prime}\right)-\tilde{\theta}(s)\right]\right. \\
& \left.\left.+\chi_{C}(s) \sin \left[\tilde{\theta}\left(s+s^{\prime}\right)-\tilde{\theta}(s)\right]\right]\right\} \\
& +\frac{K_{\rho}}{2} \bar{\rho}+\int_{0}^{\bar{\rho}} \chi_{C}(s) d s \\
\sigma_{x \theta}= & 2 K_{\theta} \frac{\partial\langle y\rangle}{\partial K_{\theta}} \\
\sigma_{y \theta}= & -2 K_{\theta} \frac{\partial\langle x\rangle}{\partial K_{\theta}}
\end{aligned}
$$

where $\chi_{C}(s)=\cos [2 \widetilde{\theta}(s)] e^{-2 K_{\theta} s}$ and $\chi_{S}(s)=\sin [2 \widetilde{\theta}(s)] e^{-2 K_{\theta^{s}}}$.

Previous equations enable us to compute the entries of the matrix $Q$ once the parameters $E_{R}, E_{T}, K_{\theta}$, and $K_{\rho}$ are known, and the trajectory $\theta(s)$ is specified. We want to remark that, in reality, the odometry sensor furnishes a discrete measurement. In other words, it provides the function $\theta(s)$ through its sampled values taken at appropriately spaced intervals. However, when the sensitivity of the odometry system is high enough, the function $\theta(s)$ can be considered provided as a continuous function and (6)-(12) can be adopted. It is possible to see that the integrals appearing in those equations can be analytically computed when the function $\theta(s)$ is linear in $s$ (i.e., circular trajectory included the case of straight trajectory; that is a special case of a circular path with infinity curvature radius).

\section{OBSERVABLES}

In this section, we introduce the observables which are measurable quantities related to a given robot motion. As we will show in Section $\mathrm{V}$, in order to more easily experimentally estimate the observables, we consider robot motions whose initial configuration coincides with the final configuration in the world coordinate frame of the odometry system.

Let us consider a given robot motion, and let us repeat this motion $n$ times. The robot motion is always the same in the world coordinate frame of the odometry system. The observables are

$$
\begin{aligned}
\operatorname{Obs}_{\theta} & =\frac{1}{n} \sum_{i=1}^{n} \Delta_{i} \\
\operatorname{Obs}_{\theta} 2 & =\frac{1}{n-1} \sum_{i=1}^{n}\left(\Delta_{i}-\operatorname{Obs}_{\theta}\right)^{2} \\
\operatorname{Obs}_{x} & =\frac{1}{n} \sum_{i=1}^{n} x_{i} \\
\operatorname{Obs}_{y} & =\frac{1}{n} \sum_{i=1}^{n} y_{i} \\
\operatorname{Obs}_{D^{2}} & =\frac{1}{n} \sum_{i=1}^{n} D_{i}^{2} \\
\operatorname{Obs}_{x \theta} & =\frac{1}{(n-1) n^{2}} \sum_{i j k}^{n}\left(x_{i} \Delta_{i}-x_{j} \Delta_{k}\right)
\end{aligned}
$$

$$
\operatorname{Obs}_{y \theta}=\frac{1}{(n-1) n^{2}} \sum_{i j k}^{n}\left(y_{i} \Delta_{i}-y_{j} \Delta_{k}\right)
$$

where $\Delta_{i}$ is the angular difference between the initial and final configurations, $x_{i}$ and $y_{i}$ are the position changes along the $\widehat{x}$ axis and $\widehat{y}$ axis, respectively, between the initial and final configurations, and $D_{i}$ is the distance between the initial and final positions related to the $i$ th robot motion.

Because of the nonsystematic errors, the observables are random variables, whose statistics are completely defined by the hypothesis introduced in Section II. In particular, on the basis of that hypothesis, it is possible to compute the mean value and the variance of each observable. We will show that the mean values are independent of the number $n$, but only depend on the considered robot motion and on the model parameters. On the other hand, their variances decrease by increasing $n$. Therefore, the observable mean value estimation is more accurate as $n$ increases.

We obtain for the mean values and variances (see [17])

$$
\left\langle\operatorname{Obs}_{\theta}\right\rangle=\overline{\Delta \theta}+E_{R} \bar{\rho}, \quad \sigma_{\mathrm{Obs}_{\theta}}=\sqrt{\frac{K_{\theta} \bar{\rho}}{n}}
$$

where $\overline{\Delta \theta}$ is the global change in orientation between the initial and final configurations measured by the encoder sensor.

$$
\begin{aligned}
\left\langle\mathrm{Obs}_{\theta}\right\rangle & =K_{\theta} \bar{\rho}, \quad \sigma_{\mathrm{Obs}_{\theta}{ }^{2}}=K_{\theta} \bar{\rho} \sqrt{\frac{2}{n-1}} \\
\left\langle\mathrm{Obs}_{x}\right\rangle & =\frac{1}{n} \sum_{i=1}^{n}\left\langle x_{i}\right\rangle=\langle x\rangle, \quad \sigma_{\mathrm{Obs}_{x}}=\frac{\sigma_{x}}{\sqrt{n}} \\
\left\langle\mathrm{Obs}_{y}\right\rangle & =\langle y\rangle, \quad \sigma_{\mathrm{Obs}_{y}}=\frac{\sigma_{y}}{\sqrt{n}}
\end{aligned}
$$

where the analytical expression of $\langle x\rangle,\langle y\rangle, \sigma_{x}$, and $\sigma_{y}$ in terms of the trajectory and the model parameters shown in Section III. Concerning the last three observables, we only give here the mean value for the sake of brevity

$$
\begin{aligned}
& \left\langle\mathrm{Obs}_{D^{2}}\right\rangle=\frac{1}{n} \sum_{i=1}^{n}\left\langle D_{i}^{2}\right\rangle=\frac{1}{n} \sum_{i=1}^{n}\left\langle x_{i}^{2}+y_{i}^{2}\right\rangle=\left\langle x^{2}\right\rangle+\left\langle y^{2}\right\rangle \\
& \left\langle\operatorname{Obs}_{x \theta}\right\rangle=\sigma_{x \theta} \quad\left\langle\operatorname{Obs}_{y \theta}\right\rangle=\sigma_{y \theta} .
\end{aligned}
$$

From (8) and (9) we therefore obtain for $\mathrm{Obs}_{D^{2}}$

$$
\begin{aligned}
\left\langle\mathrm{Obs}_{D^{2}}\right\rangle=K_{\rho} \bar{\rho}+2\left(1+E_{T}\right)^{2} \times & \int_{0}^{\bar{\rho}} d s \int_{0}^{\bar{\rho}-s} d s^{\prime}\left\{e^{-K_{\theta} s^{\prime} / 2}\right. \\
& \left.\times \cos \left[\tilde{\theta}\left(s+s^{\prime}\right)-\widetilde{\theta}(s)\right]\right\}
\end{aligned}
$$

Clearly, $\left\langle\mathrm{Obs}_{D^{2}}\right\rangle$ does not depend on the initial orientation. Moreover, this observable is very important, since it is the only one whose mean value depends on the parameter $K_{\rho}$.

Finally, concerning $\operatorname{Obs}_{x \theta}$ and $\operatorname{Obs}_{y \theta}$, their mean values can be computed through (11) and (12).

\section{Strategy to Estimate ERror Parameters}

The objective of this section is to suggest a possible strategy for estimating $E_{R}, E_{T}, K_{\theta}$, and $K_{\rho}$.

Let us consider the following simple robot motion. The robot moves straight back and forth $k$ times in order to cover a fixed distance $\bar{\rho}=$ $2 k l$ (measured by the encoder sensor). We obtain

$$
\begin{aligned}
\left\langle\mathrm{Obs}_{\theta}\right\rangle & =2 E_{R} k l, & \sigma_{\mathrm{Obs}_{\theta}} & =\sqrt{\frac{2 K_{\theta} k l}{n}} \\
\left\langle\mathrm{Obs}_{\theta}\right\rangle & =2 K_{\theta} k l, & \sigma_{\mathrm{Obs}_{\theta^{2}}} & =2 K_{\theta} k l \sqrt{\frac{2}{n-1}} .
\end{aligned}
$$


To compute the other observables for this robot motion, it is very useful to introduce the following complex quantity:

$$
z=\frac{K_{\theta} l}{2}+i E_{R} l \text {. }
$$

This complex quantity characterizes the rotational components of the odometry error. In particular, its real part contains the nonsystematic component while the imaginary part contains the systematic one.

By a direct calculation, we obtain from (22) and (23) (see Appendix II)

$$
\left\langle\mathrm{Obs}_{x}\right\rangle-i\left\langle\mathrm{Obs}_{y}\right\rangle=\left(1+E_{T}\right) l f(z)
$$

where $f(z)=\left(1-2 e^{-z}+e^{-2 z}\right)\left(e^{-2 z k}-1\right) / z\left(e^{-2 z}-1\right)$. From (30) we see that the real part of $\left(1+E_{T}\right) l f(z)$ gives the mean value $\left\langle\mathrm{Obs}_{x}\right\rangle$, and the imaginary part gives the mean value $\left\langle\mathrm{Obs}_{y}\right\rangle$.

The computation of $\left\langle\mathrm{Obs}_{D^{2}}\right\rangle$ is similar to the previous one, but a little bit more troublesome. We obtain

$$
\begin{aligned}
\left\langle\mathrm{Obs}_{D^{2}}\right\rangle= & 2 K_{\rho} k l+2\left(1+E_{T}\right)^{2} l^{2} \\
& \times \operatorname{Re}\left\{\frac{\left(e^{-z}-1\right)^{2}\left(1-e^{-2 k z}\right)+4 k\left(e^{-2 z}-1\right)}{z^{2}\left(1+e^{-z}\right)^{2}}+2 \frac{k}{z}\right\} .
\end{aligned}
$$

The mean values of the observables $\operatorname{Obs}_{x \theta}$ and $\mathrm{Obs}_{y \theta}$ can be easily obtained through differentiation using (11), (12), and (25).

Regarding the variances of the observables $\mathrm{Obs}_{x}, \mathrm{Obs}_{y}, \mathrm{Obs}_{D^{2}}$, $\mathrm{Obs}_{x \theta}$, and $\mathrm{Obs}_{y \theta}$ we need to compute $\sigma_{x}^{2}, \sigma_{x y}$, and $\sigma_{y}^{2}$ related to the considered robot motion. The computation of these quantities can be carried out starting from the general expressions given in Section III, and is similar (although a little bit more troublesome) to the computation of $\langle x\rangle-i\langle y\rangle$ given in Appendix II.

Our strategy consists of the estimation of the mean values of the observables for the considered robot motion. The advantage of this strategy is that we only need to consider the initial and final configurations of the real robot motion. In Section VI, we discuss the attainable accuracy for the parameter estimation through this strategy and we show some experimental results.

\section{DISCUSSION}

We illustrate the proposed odometry error model by discussing the attainable accuracy for the parameter estimation by adopting the proposed strategy and by showing some experimental results.

\section{A. Accuracy for the Parameters Estimation}

We discuss the attainable accuracy for the parameter estimation by adopting the proposed strategy. The error on the estimation of a given model parameter (for example $E_{R}$ ) using the observable $\mathrm{Obs}_{i}$ is given by $\Delta E_{R}=\left(\Delta\left\langle\mathrm{Obs}_{i}\right\rangle /\left|\partial\left\langle\mathrm{Obs}_{i}\right\rangle / \partial E_{R}\right|\right)=\left(\sigma_{\mathrm{Obs}_{i}} /\left|\partial\left\langle\mathrm{Obs}_{i}\right\rangle / \partial E_{R}\right|\right)$. Therefore, the relative error on the estimation of $E_{R}$ (in \%) using the observable $\mathrm{Obs}_{i}$ is given by $\left(1 / E_{R}\right)\left(\sigma_{\mathrm{Obs}_{i}} /\left|\partial\left\langle\mathrm{Obs}_{i}\right\rangle / \partial E_{R}\right|\right) \times 100$. Regarding the parameter $E_{T}$, we actually consider, as a relative error, the quantity $\left(\Delta\left(1+E_{T}\right) / 1+E_{T}\right)=\left(\Delta E_{T} / 1+E_{T}\right)$, which is much smaller than $\left(\Delta E_{T} / E_{T}\right)$.

In Table I we report the best observable to estimate each model parameter with the robot motion considered in Section V. We see that $\mathrm{Obs}_{\theta}$ and $\mathrm{Obs}_{\theta} 2$ are the best observables, respectively, to estimate $E_{R}$ and $K_{\theta}$ independently of the parameters characterizing the considered robot motion $(k, n$, and $l)$. The relative error on the estimation of these two parameters has a simple expression in terms of $k, n$, and $l$. From (27), we obtain

$$
\frac{\Delta E_{R}}{E_{R}}=\frac{1}{E_{R}} \frac{\sigma_{\mathrm{Obs}_{\theta}}}{\left|\frac{\partial\left\langle\mathrm{Obs}_{\theta}\right\rangle}{\partial E_{R}}\right|}=\frac{1}{E_{R}} \sqrt{\frac{K_{\theta}}{L_{\mathrm{Tot}}}}
$$

TABLE I

Best Observable to Estimate Each Model Parameter

\begin{tabular}{c|c|c|c}
\hline$E_{R}$ & $K_{\theta}$ & $E_{T}$ & $K_{\rho}$ \\
\hline$O b s_{\theta}$ & $O b s_{\theta^{2}}$ & $O b s_{y}, O b s_{y \theta}, O b s_{D^{2}}$ & $O b s_{D^{2}}$ \\
\hline
\end{tabular}

where $L_{\text {Tot }}=n \times 2 k l$ is the total distance traveled by the robot, and from (28), we obtain

$$
\frac{\Delta K_{\theta}}{K_{\theta}}=\frac{1}{K_{\theta}} \frac{\sigma_{\mathrm{Obs}_{\theta} 2}}{\left|\frac{\partial\left\langle\mathrm{Obs}_{\theta}{ }^{2}\right\rangle}{\partial K_{\theta}}\right|}=\sqrt{\frac{2}{n-1}} .
$$

Concerning $E_{T}$, the best observable changes by changing $k, l$, and $n$. The analytical expression of $\left(\Delta E_{T} / 1+E_{T}\right)$ using the three observables $\mathrm{Obs}_{y}, \mathrm{Obs}_{y \theta}$, and $\mathrm{Obs}_{D^{2}}$ can be obtained from the equations in Sections III-V.

In Fig. 2(a)-(c), we plot the relative error (in \%) on this parameter, versus $k$ and $n$ for a fixed $L_{\text {Tot }}$, and for the values of the model parameters we experimentally obtained in the corridor of our department (see Section VI-B). Observe that by changing these values, the qualitative behavior does not change. The relative error obtained with $\mathrm{Obs}_{y}$ [Fig. 2(a)] increases with $k$ and $n$, but the variation is in any case bounded and, in particular, does not exceed $4 \%$. Regarding the accuracy obtained through $\mathrm{Obs}_{D^{2}}$ [Fig. 2(c)], the relative error again increases with $k$ and $n$, but, in this case, the growth is unbounded. Finally, the relative error obtained by adopting $\mathrm{Obs}_{y \theta}$ [Fig. 2(b)] decreases with $n$.

Concerning $K_{\rho}$, the estimation can be obtained with good accuracy by adopting $\mathrm{Obs}_{D^{2}}$ with large $k$ and $n$ as shown in Fig. 2(d).

\section{B. Experimental Results}

1) Experiment: Our experiments consist of the estimation of the parameters $E_{R}, K_{\theta}, E_{T}$, and $K_{\rho}$ related to our mobile robot Nomad150 in two different environments, the corridor of our department (indoor) and asphalt (outdoor). Of course, it is possible to apply the same method to other mobile robots, and the results will depend not only on the robot, but also on the environment where the robot moves. In order to estimate the model parameters, we used several values of $k, n$, and $l$. In this way we could check the validity of some assumptions in Section II.

2) Robot: The Nomad150 is a three-wheeled, cylindrical, zero-gyro radius robot. Its diameter is $0.457 \mathrm{~m}$ and its height $0.406 \mathrm{~m}$. It is equipped with six sonar sensors placed at $22.5^{\circ}$ increments, which we did not use. Odometry sensors, located at the synchronous-drive system, provide an estimation of the robot's configuration. The sensitivity errors of this configuration estimation are $0.13 \mathrm{~cm}$ in the translation and $0.05^{\circ}$ in the orientation. The robot configuration is defined by the vector $X=[x, y, \theta]^{T}$ introduced in Section III. In order to evaluate the actual configuration change between the initial and final configurations in the robot motion, we fixed three screws on the base of our platform. When the robot was in the initial and final configurations, we marked the floor in correspondence with the three screws. The error associated to the distance measurement between the initial and final marks was taken equal to $0.2 \mathrm{~cm}$. In this way, it was possible to estimate the change in the orientation with an error equal to $0.3^{\circ}$ and the change in the position with an error equal to $0.1 \mathrm{~cm}$.

3) Results: We carried out four independent experiments, three in the indoor and one in the outdoor environment. In Table II, we report the parameters defining the robot motion. For each experiment, we considered appropriate values of $k$ and $n$ in order to estimate the model parameters with the best accuracy in accord with the considerations in Section VI-B.2.

Fig. 3(a) shows the observable $\mathrm{Obs}_{\theta}$ measured in the indoor environment in the three related experiments. The value of $n$ is set equal to 


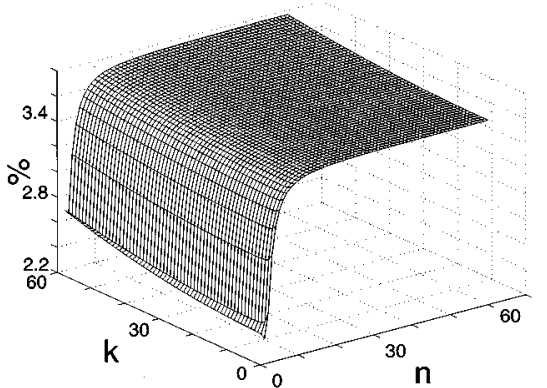

(a)

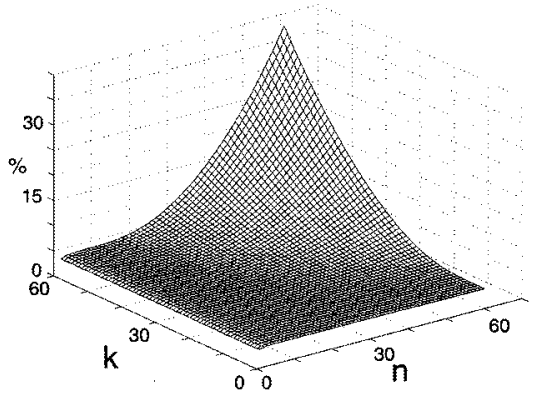

(c)

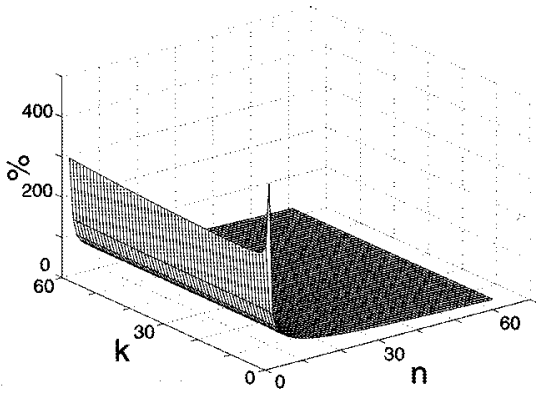

(b)

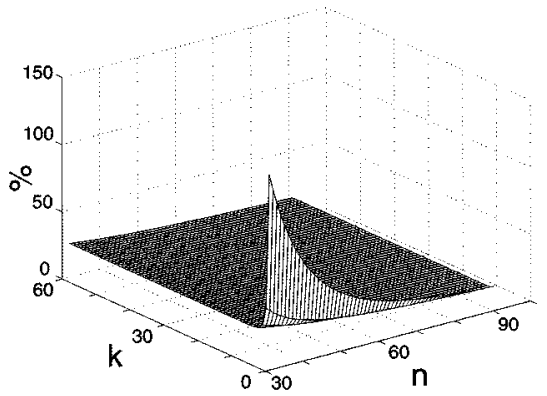

(d)

Fig. 2. Relative error on the parameter $E_{T}$ estimated by observables. (a) $\mathrm{Obs}_{y}$. (b) $\mathrm{Obs}_{y \theta}$. (c) $\mathrm{Obs}_{D^{2}}$. (d) Relative error on the parameter $K_{\rho}$ using the observable $\mathrm{Obs}_{D^{2}}$.

TABLE II

PARAMETERS DEFINING MOTION USED IN OUR EXPERIMENTS

\begin{tabular}{c|c|c|c}
\hline Experiment & $L_{T o t}(m)$ & $l(m)$ & Environment \\
\hline $1^{\text {st }}$ & 90 & 0.3 & Indoor \\
\hline $2^{\text {nd }}$ & 60 & 3.0 & Indoor \\
\hline $3^{d}$ & 180 & 6.0 & Indoor \\
\hline $4^{t h}$ & 90 & 0.3 & Outdoor \\
\hline
\end{tabular}

1 for all three results. Therefore, the corresponding $k=\left(L_{\text {Tot }} / 2 n l\right)$ are, respectively, $k=150,10$, and 15 . From this figure, we can conclude that the assumption about the linear increase of the rotational systematic component with the traveled distance is well verified. Moreover, we estimated the value $E_{R}=(-0.205 \pm 0.006)(\mathrm{deg} / \mathrm{m})$. Fig. 3(b) shows the observable $\mathrm{Obs}_{\theta}$ in the previous three experiments. Observe that in this case, the relative error decreases with $n$ (33). On the other hand, for a fixed $L_{\text {Tot }}$, we have on $n$ the constraint $\sqrt{K_{\theta} \times(2 k l)}=\sqrt{K_{\theta} \times L_{\mathrm{Tot}} / n}>\Delta \theta$, where $\Delta \theta$ is the resolution in the orientation measurement (in our case equal to $0.3^{\circ}$ ). When $\sqrt{K_{\theta} \times(2 k l)}<\Delta \theta$, it is not possible to estimate $\mathrm{Obs}_{\theta^{2}}$ ([9], [10]). Therefore, we used the following values of $n$ for the three considered experiments, $n=6,2$, and 15, respectively. From Fig. 3(b), we can again conclude that the assumption of the linear increase of the variance of the nonsystematic rotational component with the traveled distance is reasonably verified. We obtained $K_{\theta}=(0.011 \pm 0.004)\left(\mathrm{deg}^{2} / \mathrm{m}\right)$.

Concerning the estimation of $E_{T}$, we used $\mathrm{Obs}_{y}$ as suggested in Section VI-B.2. By expanding the function $f(z)$ appearing in (30), we obtain $\mathrm{Obs}_{y} \simeq-\left(1+E_{T}\right) E_{R} k l^{2}$. In Fig. 3(c), we report $\mathrm{Obs}_{y}$ versus $k l^{2} . n$ is set equal to 1 in all three considered experiments. We can again conclude that the assumption on the systematic translational component is well verified. The slope of the straight lines in the figure is $-\left(1+E_{T}\right) \times E_{R}$. Therefore, $\left(\Delta E_{T} / 1+E_{T}\right)=\left(\Delta O b_{y} / \mathrm{Obs}_{y}\right)+$ $\left(\Delta E_{R} / E_{R}\right)$. We obtained $\left(1+E_{T}\right)=0.98 \pm 0.05$.

Finally, concerning $K_{\rho}$, we could only estimate its value through the first experiment. Indeed, it is possible to see by expanding in $z$

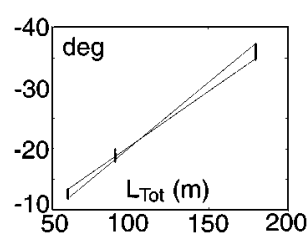

(a)

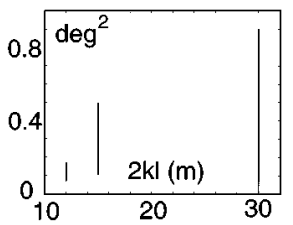

(b)

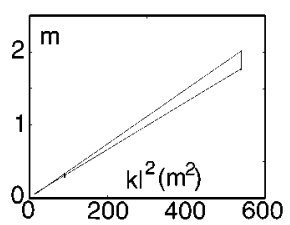

(c)

Fig. 3. Three experiments in the indoor environment. (a) $\mathrm{Obs}_{\theta}$ versus $L_{\text {Tot }}$. (b) $\mathrm{Obs}_{\theta^{2}}$ versus $2 k l$. (c) $\mathrm{Obs}_{y}$ versus $k l^{2}$.

the function appearing in (31), the second term on the right-hand side of that equation becomes much smaller than $2 K_{\rho} k l$ only when $l$ is small. In other words, the accuracy of the estimation of $K_{\rho}$ is very rough for large $l$ due to the error on the other parameters $\left(E_{R}, E_{T}\right.$ and $K_{\theta}$ ). Moreover, from Fig. 2(d), it is possible to see that the best accuracy for $K_{\rho}$ can be achieved for large $k$ and $n$. We obtained $K_{\rho}=$ $(2.2 \pm 1.0) \times 10^{-6} \mathrm{~m}$.

Concerning the outdoor environment, we found the following values through the fourth experiment: $E_{R}=(-0.25 \pm 0.04) \mathrm{deg} / \mathrm{m}, K_{\theta}=$ $(0.08 \pm 0.05)\left(\mathrm{deg}^{2} / \mathrm{m}\right),\left(1+E_{T}\right)=1.0 \pm 0.2$, and $K_{\rho}=(1.7 \pm$ 1.1) $10^{-5} \mathrm{~m}$. 


\section{CONCLUSIONS}

In this paper, we have presented a theory about the odometry error for a mobile robot with a synchronous-drive system and a strategy for evaluating the error model parameters.

The odometry error was modeled by introducing four parameters. Two parameters characterize the two nonsystematic components (translational and rotational). The other two parameters characterize the translational and rotational systematic components. The nonsystematic errors were expressed in terms of a covariance matrix, which depends both on the previous four parameters, and on the path followed by the mobile robot. We derived closed formulas for the odometry error covariance matrix, applicable to any path.

We introduced seven experimentally measurable quantities, the observables, for a given robot motion. On the basis of our odometry error model, we analytically computed the mean values and the variances of the observables, which depend on the previous four parameters and on the considered robot motion. We suggested a possible strategy for simultaneously estimating the four parameters by estimating the observables. Our strategy only required measuring the change in the orientation and the position between the initial and final configurations of the robot, related to suitable robot motions. In other words, it is unnecessary to know the actual path followed by the robot.

We illustrated the proposed strategy by discussing the accuracy of the parameters estimation and by showing some experimental results.

\section{APPENDIX I}

\section{COMPUTATION OF $\langle x\rangle$}

We want to compute the mean value of the position change along the $\widehat{x}$ axis on the basis of the odometry model discussed in Section II. We have

$$
x=\lim _{N \rightarrow \infty} x_{N}=\lim _{N \rightarrow \infty} \sum_{i=1}^{N} \widehat{\delta} \rho_{i} \cos \left(\widehat{\theta}_{i}\right)
$$

where $\widehat{\delta} \rho_{i}$ is defined in Section II, and

$$
\widehat{\theta_{i}}=\widehat{\theta}_{0}+\sum_{k=1}^{i} \widehat{\delta} \theta_{i}
$$

where $\widehat{\theta}_{0}$ is the robot orientation at the first step, and $\widehat{\delta} \theta_{i}$ is defined in Section II.

In order to compute $\langle x\rangle$, we first compute $\left\langle x_{N}\right\rangle$, and then we take the limit value when $N \rightarrow \infty$. We have

$$
\begin{aligned}
\left\langle x_{N}\right\rangle= & \int\left\{{ }_{i=1}^{N} f_{G}\left(\widehat{\delta} \rho_{1}, \sigma_{\delta \rho}\right) \cdots f_{G}\left(\widehat{\delta} \rho_{N}, \sigma_{\delta \rho}\right) f_{G}\left(\widehat{\delta} \theta_{1}, \sigma_{\delta \theta}\right) \cdots\right. \\
& \left.\quad f_{G}\left(\widehat{\delta} \theta_{N}, \sigma_{\delta \theta}\right) \sum_{i=1}^{N} \widehat{\delta} \rho_{i} \cos \left(\widehat{\theta_{i}}\right)\right\} \\
& \times d \widehat{\delta} \rho_{1} \cdots d \widehat{\delta} \rho_{N} d \widehat{\delta} \theta_{1} \cdots d \widehat{\delta} \theta_{N}
\end{aligned}
$$

where we denoted with $f_{G}\left(w, \sigma_{w}\right)$ the Gaussian distribution function of the random variable $w$, whose variance is $\sigma_{w}^{2}$.

In Section II, we assumed that $\widehat{\delta} \rho_{i}$ is independent of $\widehat{\delta} \theta_{i}$. Therefore, we obtain

$$
\left\langle x_{N}\right\rangle=\sum_{i=1}^{N}\left\langle\widehat{\delta} \rho_{i}\right\rangle\left\langle\cos \left(\widehat{\theta_{i}}\right)\right\rangle
$$

Moreover, from (1), we have

$$
\left\langle\widehat{\delta} \rho_{i}\right\rangle=\overline{\delta \rho}\left(1+E_{T}\right) .
$$

From (A2), we have

$$
\widehat{\theta_{i}} \sim N\left(\widehat{\theta_{0}}+\sum_{k=1}^{i} \overline{\delta \theta_{i}}+i E_{R} \overline{\delta_{\rho}}, K_{\theta} i \overline{\delta_{\rho}}\right)=N\left(\widetilde{\theta_{i}}, K_{\theta} i \overline{\delta_{\rho}}\right)
$$

where $\overline{\delta \theta_{i}}$ and $\overline{\delta \rho}$ are defined in Section II, and $\widetilde{\theta_{i}}$ is defined from the previous equation and represents the robot orientation at the $i$ th step as measured from the odometry sensor calibrated for the systematic errors. It is possible to obtain [17]

$$
\left\langle\cos \widehat{\theta}_{i}\right\rangle=\cos \widetilde{\theta}_{i} e^{-\left(\sigma_{\widehat{\theta}_{i}}^{2}\right) / 2}=\cos \widetilde{\theta}_{i} e^{-\left(i K_{\theta} \overline{\delta_{\rho}}\right) / 2} .
$$

Substituting (A5) and (A6) in (A4) we obtain

$$
\left\langle x_{N}\right\rangle=\left(1+E_{T}\right) \overline{\delta \rho} \sum_{i=1}^{N} \cos \left(\widetilde{\theta_{i}}\right) e^{-\left(i K_{\theta} \overline{\delta \rho}\right) / 2}
$$

and, passing to the limit $(\overline{\delta \rho}=\bar{\rho} / N)$, we obtain (6).

\section{APPENDIX II}

\section{COMPUTATION OF $\langle x\rangle-i\langle y\rangle$ FOR MOTION CONSIDERED IN SECTION V}

When the robot moves forward $\widetilde{\theta}(s)=E_{R} s$, when it moves backward $\widetilde{\theta}(s)=\pi+E_{R} s$. We therefore obtain from (6) and (7)

$$
\begin{aligned}
&\langle x\rangle=\left(1+E_{T}\right) \sum_{j=0}^{k-1} \times\left[\int_{2 j l}^{(2 j+1) l} \cos \left(E_{R} s\right) e^{-K_{\theta} s / 2} d s\right. \\
&\left. \pm \int_{(2 j+1) l}^{(2 j+2) l} \cos \left(E_{R} s\right) e^{-K_{\theta} s / 2} d s\right] \\
&\langle y\rangle=\left(1+E_{T}\right) \sum_{j=0}^{k-1} \times\left[\int_{2 j l}^{(2 j+1) l} \sin \left(E_{R} s\right) e^{-K_{\theta^{s}} / 2} d s\right. \\
&\left. \pm \int_{(2 j+1) l}^{(2 j+2) l} \sin \left(E_{R} s\right) e^{-K_{\theta} s / 2} d s\right] .
\end{aligned}
$$

Using the complex quantity defined in (29), we have

$$
\begin{aligned}
\langle x\rangle-i\langle y\rangle=\left(1+E_{T}\right) l \sum_{j=0}^{k-1} & \times\left[\int_{2 j}^{2 j+1} e^{-z s^{\prime}} d s^{\prime}\right. \\
& \left.-\int_{2 j+1}^{2 j+2} e^{-z s^{\prime}} d s^{\prime}\right] .
\end{aligned}
$$

By directly computing the integrals, we obtain the familiar geometric series $q=e^{-2 z}$ and by summing, we obtain (30).

\section{ACKNOWLEDGMENT}

The author is very grateful to S. Nicosia for the opportunity to work on this subject, and to A. Tornanbe for helpful discussions.

\section{REFERENCES}

[1] J. Borenstein, "Internal correction of dead-reckoning errors with the smart encoder trailer," in Proc. Int. Conf. Intelligent Robots and Systems, vol. 1, 1994, pp. 127-134.

[2] — "The CLAPPER: A dual-drive mobile robot with internal correction of dead-reckoning errors," in Proc. Int. Conf. Robotics and Automation, vol. 3, 1995, pp. 3085-3090.

[3] J. Borenstein and L. Feng, "Correction of systematic odometry errors in mobile robots," in Proc. Int. Conf. Intelligent Robots and Systems, vol. 3, 1995, pp. 569-574. 
[4] _ , "Measurement and correction of systematic odometry errors in mobile robots," IEEE Trans. Robot. Automat., vol. 12, pp. 869-880, Dec. 1996.

[5] — "UMBmark—A method for measuring, comparing and correcting dead-reckoning errors in mobile robots," Univ. of Michigan, Ann Arbor, Rep. UM-MEAM-94-22, Dec. 1994.

[6] J. Borenstein, "Experimental results from internal odometry error correction with the OmniMate mobile robot," IEEE Trans. Robot. Automat., vol. 14, pp. 963-969, Dec. 1998

[7] F. Chenavier and J. L. Crowley, "Position estimation for a mobile robot using vision and odometry," in Proc. Int. Conf. Robotics and Automation, vol. 3, 1992, pp. 2588-2593.

[8] K. S. Chong and L. Kleeman, "Accurate odometry and error modeling for a mobile robot," in Proc. Int. Conf. Robotics and Automation, vol. 4 1997, pp. 2783-2788.

[9] W. T. Eadie, D. Dryard, F. E. James, M. Roos, and B. Sadoulet, Statistical Method in Experimental Physics. Amsterdam, The Netherlands: North-Holland, 1971.

[10] A. G. Frodesen, O. Skjeggestad, and H. Tofte, Probability and Statistics in Particle Physics. Oslo, Norway: Universitetsforlaget, 1979.

[11] P. Goel, S. I. Roumeliotis, and G. S. Sukhatme, "Robust localization using relative and absolute position estimates," in Proc. Int. Conf. Intelligent Robots and Systems, vol. 2, 1999, pp. 1134-1140.

[12] A. Kelly, "General solution for linearized systematic error propagation in vehicle odometry," in Proc. Int. Conf. Intelligent Robot and Systems (IROSO1), Maui, HI, Oct. 29-Nov. 3 2001, pp. 1938-1945.
[13] L. Feng and E. E. Milios, "Optimal global pose estimation for consistent sensor data registration," in Proc. Int. Conf. Robotics and Automation, vol. 1, 1995, pp. 93-100.

[14] A. Martinelli, "Modeling and estimating the odometry error of a mobile robot," in Proc. 5th IFAC Symp. Nonlinear Control Systems (NOLCOSO1), St. Petersburg, Russia, July 4-6, 2001, pp. 420-425.

[15] — , "A possible strategy to estimate the odometry error of a mobile robot," in Proc. Int. Conf. Intelligent Robot and Systems (IROSO1), Maui, HI, USA, Oct. 29-Nov. 3 2001, pp. 1946-1951.

[16] — "The accuracy on the parameter estimation of an odometry system of a mobile robot," in Proc. Int. Conf. Robotics and Automation (ICRA02), Washington, DC, May 11-15, 2002, pp. 1378-1383.

[17] A. Papoulis, Probability, Random Variables, and Stochastic Process. New York: McGraw-Hill, 1991.

[18] G. J. Pappas and K. J. Kyriakopoulos, "Dynamic modeling and tracking control of nonholonomic wheeled vehicles," in Proc. IFAC'93 World Congr., 1993, pp. 241-244.

[19] N. Roy and S. Thrun, "Online self-calibration for mobile robots," in Proc. Int. Conf. Robotics and Automation, vol. 3, 1999, pp. 2292-2297.

[20] C. M. Wang, "Location estimation and uncertainty analysis for mobile robots," in Proc. Int. Conf. Robotics and Automation, 1988, pp 1231-1235.

[21] F. Zhejun, J. Borenstein, D. Wehe, and Y. Koren, "Experimental evaluation of an encoder trailer for dead-reckoning in tracked mobile robots," in Proc. IEEE Int. Symp. Intelligent Control, 1995, pp. 571-576. 